\title{
Níveis plasmáticos de homocisteína em mulheres idosas com úlceras nos pés
}

\author{
Plasma levels of homocystein in women elderly with ulcers in the foot
}

\author{
Alexandre de Souza e Silva'; José Jonas de Oliveira'; Jasiele Aparecida de Oliveira Silva²; Dayane \\ Neiry de Souza33; Maria Helena Külkamp³; Ronaldo Júlio Baganha ${ }^{1}$ \\ 1 Departamento de Educação Física do Centro Universitário de Itajubá - FEPI. Itajubá, MG - Brasil \\ 2 Departamento de Educação Física e Psicologia do Centro Universitário de Itajubá - FEPI. Itajubá, MG - Brasil \\ 3 Departamento de Podologia. Universidade Anhembi Morumbi - AM. São Paulo, SP - Brasil. \\ Endereço para Correspondência: \\ Alexandre de Souza e Silva \\ Av. Dr. Antônio Braga Filho, 687 - Bairro Varginha \\ 37.501-002 - Itajubá - MG [Brasil] \\ alexprofms@yahoo.com.br
}

\begin{abstract}
Resumo
Introdução: A homocisteína é um amino ácido produzido no fígado e seus níveis elevados apresentam relação com aterosclerose e úlceras. Objetivo: Avaliar os níveis plasmáticos de homocisteína de mulheres idosas com úlceras nos membros inferiores. Métodos: Participaram 40 mulheres idosas, idade média 67,4 $\pm 6,49$ anos, divididas em grupo com úlcera (GU) e controle (GC). A avaliação da presença de úlcera foi realizada por observação, a avaliação da concentração de homocisteína $(\mu \mathrm{mol} / \mathrm{L})$ foi realizada por high performance liquid chromatography (HPLC) e a aferição da pressão arterial foi realizada pelo uso de um esfigmomanômetro aneroide - HICO HM 1001. Resultados: Níveis de homocisteína mais elevados para o grupo úlcera $17,69 \pm 6,82$ versus controle $11,70 \pm 1,51$. As pressões arteriais sistólica e diastólica não apresentaram diferenças entre os grupos ulcera $134,16 \pm 19,28 / 74,16 \pm 11,64$ e controle $136,15 \pm 16,09 / 77,69 \pm 5,99$ respectivamente. Conclusão: Conclui-se que os níveis plasmáticos de homocisteína em mulheres idosas com úlcera estão aumentados.
\end{abstract}

Descritores: Hiper-Homocisteinemia; Doenças Cardiovasculares; Aterosclerose.

\begin{abstract}
Introduction: Homocysteine is an amino acid produced in the liver and its elevated levels are related to atherosclerosis and ulcer. Objective: To evaluate the plasma levels of homocysteine of elderly women with ulcers in the lower limbs. Methods: Participated 40 elderly women, mean age $67.4 \pm 6.49$ years, separated into ulcer (GU) and control (CG) groups. The evaluation of the presence of ulcer was performed by observation, the homocysteine concentration $(\mu \mathrm{mol} / \mathrm{L})$ was evaluated by high performance liquid chromatography (HPLC) and blood pressure was measured using an aneroid sphygmomanometer HICO HM 1001. Results: Higher homocysteine levels for the ulcer group $17.69 \pm 6.82$ vs control $11.70 \pm$ 1.51. The systolic and diastolic blood pressures did not differences between the ulcer group $134,16 \pm 19,28 / 74,16 \pm 11,64$ and control group $136,15 \pm 16,09 / 77,69 \pm 5,99$, respectively. Conclusion: It is concluded that plasma levels of homocysteine in elderly women with ulcer are increased.
\end{abstract}

Key words: Hyperhomocysteinemia; Cardiovascular diseases; Atherosclerosis. 


\section{Introdução}

A homocisteína é um aminoácido produzido no fígado por desmetilação e/ou transulfuração $0^{1,2}$, sendo a hiperhomocisteínemia um dos principais fatores de riscos para desenvolvimento de doenças cardiovasculares como a aterosclerose.

A aterosclerose é caracterizada pela formação de placas de ateroma nos vasos sanguíneos $^{3}$, o que resulta em elevação da resistência vascular periférica, redução da plasticidade vascular $^{2}$ e do fluxo sanguíneo, principalmente nos membros inferiores ${ }^{4}$.

A estenose vascular por aterosclerose reduz a oferta de nutrientes, oxigênio e remoção de subprodutos do metabolismo, sendo estas condições relacionadas à redução da capacidade regenerativa ${ }^{5}$, necrose celular e amputação de membro $^{6}$, proliferação bacteriana ${ }^{7}$ e aparecimento de úlceras ${ }^{8}$. A hiperhomocisteinemia potencializa a instalação da aterosclerose e estenose vascular ${ }^{9,10}$, sendo ambas as condições relacionadas ao aparecimento de úlceras.

Devido à elevada prevalência da aterosclerose em idosos e sua relação com a hiperhomocisteinemia, a avaliação da concentração plasmática da homocisteína pode servir de prognóstico ao aparecimento de úlceras ${ }^{6}$. A hipótese do presente estudo é que indivíduos com úlceras nos pés apresentam níveis plasmáticos de homocisteína mais elevados se comparados a indivíduos sem ulcera. O objetivo do estudo foi avaliar as concentrações plasmáticas de homocisteína de mulheres idosas com úlceras nos pés.

\section{Materiais e métodos}

\section{Participantes}

Foram selecionadas 40 mulheres idosas,

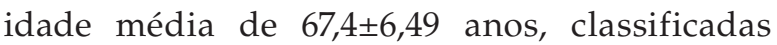
como inativas, portadoras de diabetes mellitus tipo II, com sobrepeso e classificadas como normotensas de acordo com a VII Diretriz Brasileira de Hipertensão ${ }^{11}$, com e sem a presença de ul- ceras pés. Todas as voluntárias faziam uso de hipoglicemiante oral. As voluntárias foram selecionadas a partir de contato direto com as mesmas em um centro de $3^{\text {a }}$ idade localizado em uma cidade do sul de Minas Gerais. Foram inclusas voluntárias idosas com e sem a presença de úlceras nos membros inferiores, normotensas, que concordaram com todos os procedimentos adotados no estudo. Todas as voluntárias inclusas assinaram o TCLE. O presente estudo foi aprovado pelo Comitê de Ética e Pesquisa da Universidade Anhembi Morumbi, sob o protocolo $\mathrm{n}^{\mathrm{o}}$ 0212.0.201.000-11.

As voluntárias foram divididas em dois grupos, grupo GU (grupo úlcera) e GC (grupo controle), sendo cada um composto por $20 \mathrm{mu}$ lheres idosas (figura 1).

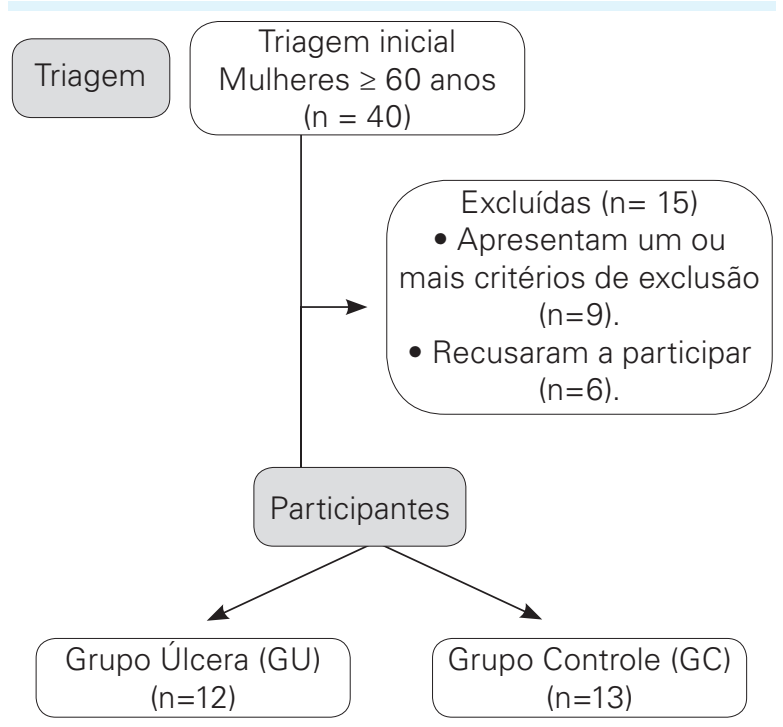

Figura 1: Fluxograma das etapas de seleção, inclusão e exclusão das idosas participantes do estudo Fonte: Próprio autor.

\section{Procedimentos}

Em dia e hora marcado com todas as voluntárias, as mesmas passaram por uma avaliação física na qual foi analisada a massa corporal $(\mathrm{Kg})$ em balança Filizola ${ }^{\circledR}$ com capacidade de $180 \mathrm{~kg}$ e precisão de 100 gramas, a estatura pelo uso de um estadiômetro (Seca ${ }^{\circledR}$, Alemanha), 
com escala de 0 a $220 \mathrm{~cm}$ e precisão de $0,1 \mathrm{~cm}$ e foi feito o calculo do índice de massa corporal $\left(\mathrm{IMC} \mathrm{Kg} / \mathrm{m}^{2}\right)^{12}$.

Após a avaliação física, as voluntárias foram submetidas a uma coleta de sangue realizada na fossa antecubital do braço não dominante pelo uso de tubos a vácuo com capacidade de 4 $\mathrm{ml}$, contendo anticoagulante EDTA (ácido etilenodiaminotetraacético).

Após a coleta, o material biológico foi centrifugado em centrífuga Eppendor ${ }^{\circledR}$ a $1200 \mathrm{rpm} /$ minuto, durante 20 minutos, sendo o plasma foi separado e congelado a menos $20^{\circ} \mathrm{C}$ para posterior análise. Por ocasião da coleta de sangue, as voluntárias estavam em jejum de 12 horas.

A análise dos níveis plasmáticos de homocisteína aconteceu pelo método de cromatografia líquida de alta performance (HPLC), com uso do reagente da DPC-Medlab, tendo como intervalo de normalidade valores entre 5,0 e 15,0 micromóis/L e sensibilidade analítica de 0,5 micromóis/L $\mathrm{L}^{13,14,15}$.

A pressão arterial sistólica e diastólica em repouso foi mensurada por meio de um esfigmomanômetro aneroide - HICO HM 1001, devidamente calibrado. As medidas foram realizadas em triplicatas no braço esquerdo das idosas, seguindo as recomendações da sociedade brasileira de cardiologia ${ }^{11}$, sendo considerado para análise o valor médio entre as três medidas.

Para o diagnóstico das úlceras, levou-se em consideração os fatores: profundidade, extensão, localização, odor, temperatura, aparência, exsudato, pele brilhante e presença de infecção, de acordo com os critérios estabelecidos, sendo feito por meio de observação destes sinais ${ }^{16}$.

\section{Anólise estatística}

Inicialmente foi avaliada a normalidade dos dados pelo teste de Shapiro-Wilk, sendo os mesmos classificados como paramétricos, utilizou se o teste $t$ de Student. Foi calculada também a variação percentual entre os grupos $(\Delta \%)$ e analisado a tendência dos dados, pelo uso da formula de Cohen para effect size ${ }^{17}$. O limiar de magnitude adotado foi: $\leq 0,19$ trivial; entre 0,20-0,59 pequeno; entre 0,60-1,19 moderado; entre 1,20-1,99 grande; e $\geq 2,00$ muito grande ${ }^{18}$. O software utilizado para análise dos dados foi o SPSS Statistics 20.0® e o nível de significância adotado para todas as situações foi de $p \leq 0,05$.

\section{Resultados}

Finalizaram o presente estudo 23 idosas, sendo 12 no GU e 13 no GC. A tabela 1 apresenta a caracterização da amostra. Não foi observado diferença significativa para as variáveis idade (anos), massa corporal (Kg), altura (metros) e $\operatorname{IMC}\left(\mathrm{kg} / \mathrm{m}^{2}\right)(\mathrm{p} \leq 5 \%)$.

A tabela 2 apresenta a comparação da concentração plasmática da homocisteína e pressão arterial das voluntárias dos grupos GU e GC. Pode ser observado que o GU possui concentração de homocisteína mais elevado em relação ao GC ( $\mathrm{p} \leq 5 \%)$. A PA não apresentou diferenças significativas entre os grupos.

Tabela 1: Características dos grupos com e sem úlceras nos pés

\begin{tabular}{ccccc}
\hline & $\begin{array}{c}\text { Grupo Úlcera } \\
\text { (GU) }\end{array}$ & $\begin{array}{c}\text { Grupo } \\
\text { Controle (GC) }\end{array}$ & $\Delta \%$ & $p$ \\
\hline $\begin{array}{c}\text { Idade } \\
\text { (anos) }\end{array}$ & $65,66 \pm 5,78$ & $69,15 \pm 7,20$ & 5,32 & 0,19 \\
\hline $\begin{array}{c}\text { Massa } \\
\text { (Kg) }\end{array}$ & $86,14 \pm 23,69$ & $72,30 \pm 12,04$ & 16,07 & 0,07 \\
\hline $\begin{array}{c}\text { Altura } \\
(\mathrm{m})\end{array}$ & $1,61 \pm 0,06$ & $1,56 \pm 0,05$ & 3,11 & 0,05 \\
\hline $\begin{array}{c}\text { IMC } \\
\left(\mathrm{Kg} / \mathrm{m}^{2}\right)\end{array}$ & $33,14 \pm 8,69$ & $29,50 \pm 5,27$ & 10,98 & 0,22 \\
\hline
\end{tabular}

Valores apresentados em média e desvio padrão. $\mathrm{Kg}=$ quilograma. $\mathrm{M}=$ metro. $\mathrm{IMC}=$ Índice de massa corporal.

Fonte: Próprio autor.

\section{Discussão}

O objetivo do estudo foi avaliar os níveis plasmáticos de homocisteína em mulheres idosas com úlceras nos pés. O principal achado do presente estudo foi à verificação de que idosas 
Tabela 2: Comparação das variớveis entre o grupo com úlcera e o controle

\begin{tabular}{cccccc}
\hline & Grupo Úlcera & Grupo Controle & $\Delta \%$ & Effect Size & $p$ \\
\hline Homocisteína $(\mu \mathrm{mol} / \mathrm{L})$ & $17,69 \pm 6,82$ & $11,70 \pm 1,51^{*}$ & 33,86 & 0,51 (pequeno) & 0,00 \\
\hline PAS $(\mathrm{mmHg})$ & $134,16 \pm 19,28$ & $136,15 \pm 16,09$ & 1,48 & $-0,05$ (trivial) & 0,78 \\
\hline PAD $(\mathrm{mmHg})$ & $74,16 \pm 11,64$ & $77,69 \pm 5,99$ & 4,76 & $-0,18$ (trivial) & 0,34 \\
\hline
\end{tabular}

Valores apresentados em média e desvio padrão.

PAS. Pressão arterial sistólica. PAD. Pressão arterial diastólica. * $(p \leq 0,05)$

Fonte: Próprio autor.

do GU nos pés possuem níveis plasmáticos de homocisteína mais elevados em relação ao GC, assim, a hipótese inicial foi confirmada.

Corroborando com nossos dados, González et al. ${ }^{4}$, avaliou pacientes com idade igual ou superior a 40 anos e os resultados apresentaram níveis elevados de homocisteína no grupo úlcera quando comparado ao grupo controle. A hiperhomocisteinemia associa-se com a formação de tromboembolismo arterial e venoso e aterosclerose ${ }^{19,20}$. A homocisteína pode estimular a oxidação da lipoproteína de baixa densidade (LDL), sendo esta condição associada a lesões endoteliais, trombogênese e aterogênese ${ }^{2,8}$.

A hiperhomocisteínemia é estabelecida como um fator de risco para doenças cardiovasculares $^{9,20}$, demonstrando associação com as doenças vasculares periféricas, o que suporta a hipótese de que níveis elevados de homocisteína podem ser um marcador/indicador de alterações vasculares ${ }^{6,21}$.

Dentre as feridas crônicas mais comuns nos membros inferiores, pode-se citar a ulcera dos pés ${ }^{5}$, sendo as crônicas com prevalência de $1 \%$ na população adulta e de 3 a $5 \%$ na população idosa, podendo levar há uma redução da qualidade de vida e diminuição das atividades diárias ${ }^{6,22,23}$.

A hiperhomocisteínemia é aceita como um fator de risco independente para trombose venosa profunda, sendo possível e plausível que a mesma contribuía para o desenvolvimento de úlceras nos pés ${ }^{6}$.

Bhargava et al. ${ }^{24}$ demonstraram que mais da metade dos pacientes com estenose arterial apresentam hiperhomocisteínemia. Segundo
Fermo et al. ${ }^{25}$, a hiperhomocisteínemia demonstrou relação positiva com as doenças vasculares oclusivas prematuras em pacientes, sendo assim, a hiperhomocisteinemia pode ser um marcador de feridas nos membros inferiores ${ }^{6}$.

No presente estudo não foi observado diferença significativa na pressão arterial sistólica e diastólica. A elevação da pressão arterial está diretamente ligada a problemas vasculares ${ }^{26,27}$, no entanto, os valores pressóricos encontrados (tabela 2) estão dentro dos valores de normalidade em ambos os grupos ${ }^{11}$. Como a hiperhomocisteínemia é um coadjuvante na elevação da pressão arterial, o controle dessa variável é importante para manutenção da saúde de idosos.

\section{Conclusão}

Conclui-se com o presente estudo que os níveis plasmáticos de homocisteína estão aumentados em mulheres idosas com úlcera nos membros inferiores. Sugere-se a realização de mais estudos prospectivos para elucidar o real papel da hiperhomocisteinemia plasmática no desenvolvimento da úlcera nos pés de idosas.

\section{Referências}

1. Brustolin S, Giugliani R, Félix TM. Genetics of homocysteine metabolism and associated disorders. Braz J Med Biol Res. 2010;43(1):1-7.

2. Neves LB, Macedo DM, Lopes AC. Homocisteína. J Bras Patol Med Lab. 2004;40(5):311-320. 
3. Kim MS, Kang SJ, Lee CW, Han S, Park DW, Lee SW, et al. Prevalence of Coronary Atherosclerosis in Asymptomatic Healthy Subjects: An Intravascular Ultrasound Study of Donor Hearts. J Atheroscler Thromb. 2013;20(5):1-7.

4. González R, Pedro T, Real JT, Martínez-Hervás S, Abellán MR, Lorente $\mathrm{R}$, et al. Plasma homocysteine levels are associated with ulceration of the foot in patients with type 2 diabetes mellitus. Diabetes Metab Res Rev. 2010;26(2):115-120.

5. Spentzouris S, Labropoulos N. The Evaluation of Lower-Extremity Ulcers. Semin Intervent Radiol. 2009;26(4):286-295.

6. Schwartzfarb EM, Romanelli P. Hyperhomocysteinemia and lower extremity wounds. Int J Low Extrem Wounds. 2008;7(3):126-136

7. Hatunic M, Finucane F, Burns N, Gasparro D, Nolan JJ. Vascular inflammatory markers in early-onset obese and type 2 diabetes subjects before and after three months' aerobic exercise training. Diab Vasc Dis Res. 2007;4(3):231-234.

8. Martí-Carvajal AJ, Sola I, Lathyris D, Salanti G. Homocysteine-Lowering Interventions for Preventing Cardiovascular Events. Cochrane Database Syst Rev. 2013;1.

9. Peng HY, Man CF, Xu J, Fan Y. Elevated homocysteine levels and risk of cardiovascular and all-cause mortality: a meta-analysis of prospective studies. J Zhejiang Univ Sci B. 2015;16(1):78-86.

10. Brustolin S, Giugliani R, Félix TM. Genetics of homocysteine metabolism and associated disorders. Braz J Med Biol Res. 2010;43(1):1-7.

11. Sociedade Brasileira de Cardiologia. Sociedade Brasileira de Hipertensão. Sociedade Brasileira de Nefrologia. VI Diretrizes Brasileiras de Hipertensão. Arq Bras Cardiol. 2017; 107(3): 1-103.

12. Deurenberg-Yap M, Schmidt G, van Staveren WA, Deurenberg $P$. The paradox of low body mass index and high body fat percentage among Chinese, Malays and Indians in Singapore. International Int J Obes Relat Metab Disord. 2000;24(8):1011-1017.

13. Ichinose S, Nakamura M, Maeda M, Ikeda R, Wada M, Nakazato M, et al. A validated HPLCfluorescence method with a semi-micro column for routine determination of homocysteine, cysteine and cysteamine, and the relation between the thiol derivatives in normal human plasma. Biomed Chromatogr. 2009;23(9):935-939.
14. Ferin R, Pavão ML, Baptista J. Methodology for a rapid and simultaneous determination of total cysteine, homocysteine, cysteinylglycine and glutathione in plasma by isocratic RP-HPLC. J Chromatogr B Analyt Technol Biomed Life Sci. 2012;911:15-20.

15. Sawuta JP, Banecka-Majkutewicz Z, Kadzinski L, Jakóbkiewicz-Banecka J, Wegrzyn G, Nyka W, et al. Improved HPLC method for total plasma homocysteine detection and quantification. Acta Biochim Pol. 2008;55(1):119-125.

16. Wagner FWJ. The dysvascular foot: a systemof diagnosis and treatment. Foot Ankle. 1981;2(2):64-122.

17. Cohen J. Statistical Power Analysis for the Behavioral Sciences. New York: Routledge Academic; 1988.

18. Hopkins GW, Marshall WS, Batterham MA, Hanin J. Progressive Statistics for Studies in Sports Medicine and Exercise Science. Med Sci Sports Exerc. 2009;41(1):3-12.

19. Kumar J, Ingelsson E, Lind L, Fall T. No evidence of a causal relationship between plasma homocysteine and type 2 diabetes: a Mendelian randomization study. Front Cardiovasc Med. 2015;2:1-6.

20. Wang W, Gao C, Yu C, Liu S, Hou D, Wang, Y, et al. No Association between elevated total homocysteine levels and functional outcome in elderly patients with acute cerebral infarction. Front Aging Neurosci. 2017;9:1-7.

21. Salagre E, Vizuete AF, Leite M, Brownstein DJ McGuinness A, Jacka F, et al. Homocysteine as a peripheral biomarker in bipolar disorder: a metaanalysis. Eur Psychiatry. 2017;43:81-91.

22. Kadia BM, Dimala CA, Aroke D, Ekabe CJ, Kadia RS, Mefire AC. A prospective analysis of pinch grafting of chronic leg ulcers in a series of elderly patients in rural Cameroon. BMC Dermatol. 2017;43(1):81-91.

23. Brod M. Quality of life issues in patients with diabetes and lower extremity ulcers: patients and care givers. Qual Life Res. 1998;7(4):365-372.

24. Bhargava S, Parakh R, Manocha A, Ali A, Srivastava LM. Prevalence of hyperhomocysteinemia in vascular disease: comparative study of thrombotic venous disease vis-a-vis occlusive arterial disease. Vascular. 2007;15(3):149-153. 
25. Fermo I, Vigano'D'Angelo S, Paroni R, Mazzola G, Calori G, D'Angelo A. Prevalence of moderate hyperhomocysteinemia in patients with early-onset venous and arterial occlusive disease. Ann Intern Med. 1995;123(10):747-753.

26. Zhang Z, Fang X, Hua Y, Liu B, Ji X, Tang Z, et al. Combined effect of hyperhomocysteinemia and hypertension on the presence of early carotid artery atherosclerosis. J Stroke Cerebrovasc Dis. 2016;25(25):1254-1262.
27. Zhong F, Zhuang L, Wang Y, Ma Y. Homocysteine levels and risk of essential hypertension: A metaanalysis of published epidemiological studies. Clin Exp Hypertens. 2017;39(2):160-167. 